\title{
Returning from Oblivion: Imaging the Neural Core of Consciousness
}

\author{
Jaakko W. Långsjö, ${ }^{1,2 \star}$ Michael T. Alkire, ${ }^{3 \star}$ Kimmo Kaskinoro, ${ }^{4}$ Hiroki Hayama, ${ }^{3}$ Anu Maksimow, ${ }^{4}$ Kaike K. Kaisti, ${ }^{1}$ \\ Sargo Aalto, ${ }^{1}$ Riku Aantaa, ${ }^{4}$ Satu K. Jääskeläinen, ${ }^{5}$ Antti Revonsuo, ${ }^{6}$ and Harry Scheinin ${ }^{1,7,8}$ \\ ${ }^{1}$ Turku PET Centre, University of Turku and Turku University Hospital, FI-20521 Turku, Finland, ${ }^{2}$ Department of Anesthesiology, Seinäjoki Central \\ Hospital, 60220 Seinäjoki, Finland, ${ }^{3}$ VA Long Beach Healthcare system (Long Beach, California, 90822), Department of Anesthesiology \& Perioperative Care \\ and the Center for the Neurobiology of Learning and Memory, University of California-Irvine, California 92868, ${ }^{4}$ Department of Anesthesiology, Intensive \\ Care, Emergency Care, and Pain Medicine, Turku University Hospital, FI-20521 Turku, Finland, ${ }^{5}$ Department of Clinical Neurophysiology, Turku \\ University Hospital, FI-20521 Turku, Finland, ${ }^{\circ}$ Department of Psychology, University of Turku, FI-20014 Turku, Finland and School of Humanities and \\ Informatics, University of Skövde, Skövde, Sweden, ${ }^{7}$ Department of Pharmacology, Drug Development and Therapeutics, University of Turku, FI-20014 \\ Turku, Finland, ${ }^{8}$ Pulssi Medical Centre, 20100 Turku, Finland
}

One of the greatest challenges of modern neuroscience is to discover the neural mechanisms of consciousness and to explain how they produce the conscious state. We sought the underlying neural substrate of human consciousness by manipulating the level of consciousness in volunteers with anesthetic agents and visualizing the resultant changes in brain activity using regional cerebral blood flow imaging with positron emission tomography. Study design and methodology were chosen to dissociate the state-related changes in consciousness from the effects of the anesthetic drugs. We found the emergence of consciousness, as assessed with a motor response to a spoken command, to be associated with the activation of a core network involving subcortical and limbic regions that become functionally coupled with parts of frontal and inferior parietal cortices upon awakening from unconsciousness. The neural core of consciousness thus involves forebrain arousal acting to link motor intentions originating in posterior sensory integration regions with motor action control arising in more anterior brain regions. These findings reveal the clearest picture yet of the minimal neural correlates required for a conscious state to emerge.

\section{Introduction}

Consciousness is a central question in neuroscience (Crick and Koch, 2003). Yet, the neural correlates of consciousness remain poorly understood. Consciousness research can roughly be divided into two categories: (1) studies on the contents of consciousness and (2) studies on consciousness as a state (Dehaene and Changeux, 2011). Whereas theories on the particular contents of consciousness, such as visual consciousness, argue for the importance of cortical structures (Lamme, 2006), theories focusing on consciousness as a state stress the importance of subcortical (Baars, 1995; Fuller et al., 2011) or thalamocortical structures (Tononi, 2004; Merker, 2007). Having awareness of the environment or of one's self is fundamentally based on being in a con-

\footnotetext{
Received Sept. 29, 2011; revised Jan. 25, 2012; accepted Feb. 16, 2012.

Author contributions: J.W.L., K.K., A.M., K.K.K., R.A., S.K.J., and H.S. designed research; J.W.L., K.K., A.M., K.K.K., and H.S. performed research; M.T.A., H.H., and S.A. analyzed data; J.W.L., M.T.A., A.R., and H.S. wrote the paper.

This work was funded by the Academy of Finland (Research Programme on Neuroscience, NEURO, project no. 8111818), Turku University Hospital EV0-Grant no. 13323, Seinäjoki Central Hospital EV0-Grant no. 1133, The Finnish Medical Foundation, and The Paulo Foundation. Presented in part at the American Society of Anesthesiologist's annual meeting, Best abstracts of the meeting session, 0ctober 16-20, 2010, San Diego, California.

*J.W.L. and M.T.A. contributed equally to this work.

The authors declare no competing financial interests.

This article is freely available online through the J Neurosci Open Choice option.

Correspondence should be addressed to Harry Scheinin, Turku PET Centre, University of Turku, and Turku University Hospital, P.0. Box 52, FI-20521 Turku, Finland. E-mail: harry.scheinin@utu.fi.

DOI:10.1523/JNEUROSCI.4962-11.2012

Copyright $\odot 2012$ the authors $\quad 0270-6474 / 12 / 324935-09 \$ 15.00 / 0$
}

scious state (Fig. 1). There is limited human data on which brain structures engage to serve this foundation of consciousness. This study was designed to reveal the minimal neural correlates associated with a conscious state.

Anesthesia is a tool for studying consciousness as it allows the level of consciousness to be directly manipulated (Beecher, 1947; Alkire et al., 2008; Stamatakis et al., 2010; Schrouff et al., 2011; Xie et al., 2011). However, use of this tool requires overcoming the limitation that a drug-induced unconscious state is necessarily confounded by the presence of the drug used to induce unconsciousness (Tononi and Koch, 2008). To illustrate, anesthesia may reduce neuronal activity throughout the brain, yet only its actions in select areas might be directly related to its ability to induce unconsciousness (Franks, 2008). Also, due to differences in hypnotic mechanisms among anesthetic agents (Alkire et al., 2008; Brown et al., 2010), studying only one drug will make generalizations problematic.

In the present study, we overcame these limitations in three distinct ways. First, we used neuroimaging in conjunction with pattern analysis methodology to dissociate the state-related changes in consciousness from the global effects of anesthesia (McIntosh and Lobaugh, 2004). Second, we eliminated the drugdose change effect on consciousness by imaging a rapid return to consciousness from the unconscious state induced by the unique anesthetic agent dexmedetomidine, a selective $\alpha$-2-adrenergic agonist, which allows awakening during constant dosing of the drug (Maksimow et al., 2007). Third, with neuroimaging we dis- 


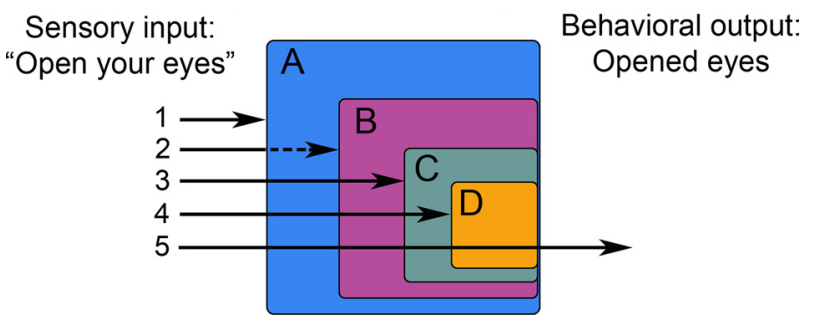

Figure 1. Conscious responsiveness schematic. Sensory input results in purposeful behavioral output only when all aspects of conscious information processing $(\boldsymbol{A}$, conscious state; $\boldsymbol{B}$, awareness and comprehension of the stimulus) and motor readiness ( $\boldsymbol{C}$, will and intention to respond; $\boldsymbol{D}$, ability to respond) are functional. Arrows represent signal processing in different consciousness states: 1 , all stimuli blocked from reaching consciousness (deep anesthesia or brain death); 2, dreaming during sleep or anesthesia, surrounding stimuli may (but not necessarily) (slashed arrow) influence dream content; 3 , deficient will to act (e.g., anterior cingulate lesion) (Crick, 1994); 4, inability to respond regardless of total awareness (e.g., awareness during anesthesia); 5, commands lead to purposeful responses (normal waking consciousness).

sociated the drug-specific effects on consciousness by showing similar activation to arousal from anesthesia with two different drugs. With these approaches we were able to reveal the minimal neural correlates for arousal into a conscious state.

The ability to rapidly and repeatedly change a person's state of consciousness with anesthesia is limited by the pharmacodynamics of anesthetic drugs. Whereas people can be quickly rendered unconscious with anesthesia, the emergence from anesthesia is often variable (Friedman et al., 2010). Thus, imaging techniques, such as functional magnetic resonance imaging, that must overcome their inherent signal-to-noise problems by using multiple behavioral transitions occurring in a relatively short time frame will have limited utility for imaging the changes in consciousness caused by anesthesia. Here, we used positron emission tomography (PET) to image the brain activity changes associated with varying states of consciousness.

\section{Materials and Methods}

Participants

After obtaining written informed consents and the approvals of the Ethical Committee of the Hospital District of Southwest Finland (Turku, Finland) and the National Agency for Medicines, 20 healthy, right-handed male volunteers (19-28 years) participated in this open, nonrandomized study. All subjects abstained from alcohol use or medication for $48 \mathrm{~h}$ and fasted overnight before the study sessions. No premedication was given.

\section{Monitoring}

A right forearm vein was cannulated for administration of the anesthetic agents and $\left[{ }^{15} \mathrm{O}\right] \mathrm{H}_{2} \mathrm{O}$. Ringer's acetate infusion was used to keep the intravenous catheter open. The left radial artery was cannulated for arterial blood activity measurements. A GE Datex-Ohmeda S/5 anesthesia monitor (GE Healthcare) was used to record subjects' noninvasive blood pressure, oxygen saturation, electrocardiogram, and the inspiratory and expiratory gas levels of oxygen and carbon dioxide throughout the study. Bispectral index and spectral entropy values were recorded with disposable electroencephalograph (EEG) electrode strips placed on each subject's forehead; the results of which have been presented previously (Kaskinoro et al., 2011).

\section{Anesthesia sessions}

General anesthesia is often defined as comprising pharmacologically induced unconsciousness (loss of the ability to feel or experience anything), amnesia (forgetfulness), analgesia (lack of pain), and immobility (not moving in response to surgical stimulation) (Brown et al., 2010). In this study, without surgical stimulation, the term "anesthesia" is used to describe pharmacologically induced loss of consciousness (LOC). Its objective behavioral criterion was unresponsiveness to a verbal command.

Subjects were divided into two groups to receive either dexmedetomidine $(n=10)$ or propofol $(n=10)$ via computer-driven target- controlled infusion (TCI). Due to wide individual variability in the anesthetic dose required for LOC, scanning subjects on a fixed dose of an agent would place them in different behavioral states. To account for this, each subject's individual concentration for LOC was first determined in a separate study session (Session 1 ) one month prior to the neuroimaging (Session 2). The individual doses determined in Session 1 were then used to guide drug dosing during neuroimaging in Session 2. Once a subject was assigned for a particular anesthetic agent in Session 1, that subject then again received that anesthetic during neuroimaging in Session 2.

Dexmedetomidine (Precedex $100 \mu \mathrm{g} / \mathrm{ml}$; Orion). This was administered via TCI. A Harvard Model 22 syringe pump (Harvard Apparatus) connected to a portable computer running STANPUMP software (by Steven L. Schafer, $\mathrm{MD}$, available at http://www.opentci.org/doku.php?id=code:code) and the pharmacokinetic parameters of Talke (2003) were used. In Session 1, the infusion was started at a target (plasma) concentration of $1.0 \mathrm{ng} / \mathrm{ml}$, followed first by a $0.5 \mathrm{ng} / \mathrm{ml}$ target concentration increase, and then by subsequent $0.25 \mathrm{ng} / \mathrm{ml}$ increases thereafter (i.e., 1.0 to $1.5,1.5$ to $1.75,1.75$ to 2.0 , and 2.0 to $2.25 \mathrm{ng} / \mathrm{ml}$ ) until LOC was achieved.

Propofol. Propofol Lipuro $10 \mathrm{mg} / \mathrm{ml}$ (B. Braun) was administered via TCI using the Marsh pharmacokinetic model (Marsh et al., 1991). In Session 1, the infusion was started at a target (plasma) concentration of $1.0 \mu \mathrm{g} / \mathrm{ml}$, followed first by a $0.5 \mu \mathrm{g} / \mathrm{ml}$ target concentration increase, and then subsequent $0.25 \mu \mathrm{g} / \mathrm{ml}$ increases thereafter until LOC was achieved.

In Session 1, infusions were started at a low rate and then increased in a stepwise manner at $10 \mathrm{~min}$ intervals until LOC. Consciousness was tested at $5 \mathrm{~min}$ intervals by asking the subjects to open their eyes. LOC was defined as being unresponsive to this request. In Session 2, neuroimaging data were obtained during four different constant states of consciousness induced with either dexmedetomidine or propofol. Subjects were scanned (1) at baseline (BL), (2) during sedation (Sed), (3) upon LOC, and (4) upon the return of consciousness (ROC) during the emergence from the anesthesia. To achieve these behavioral states, the drug infusions were aimed at 50,75 , and $100 \%$ of the individually predetermined doses for LOC. Consciousness was tested once during each concentration level, i.e., $8 \mathrm{~min}$ after the infusion was started or increased. Scanning was started $2 \mathrm{~min}$ after the consciousness testing, and no testing was performed during neuroimaging. If the LOC concentration defined in Session 1 was insufficient to produce LOC in Session 2, the drug infusion was increased an additional $25 \%$ and scanning was repeated. Infusion rates were adjusted upward at $12 \mathrm{~min}$ intervals until the LOC condition was reached and imaged. A sedative state was defined as the last testing condition before LOC where subjects could still open their eyes. During both sessions, subjects were instructed to keep their eyes closed unless specifically asked to open them.

After the LOC scan was completed, consciousness for those in the dexmedetomidine group was restored using gentle tactile and verbal stimulation while the subjects were still receiving an uninterrupted continuous steady drug infusion. After subjects regained their consciousness, a scan for ROC condition was performed. Subsequently, an additional scan (LOC-2) was performed when these subjects again lost consciousness after they were left unstimulated in the scanner for a few minutes. For those in the propofol group, the infusion was terminated and the subjects were allowed to emerge from the anesthesia. Consciousness was tested at $1 \mathrm{~min}$ intervals until ROC. When a conscious state had returned, the subjects were scanned in the ROC condition. ROC was defined as the first eye opening in response to command after LOC.

\section{Drug assays}

Plasma concentrations of dexmedetomidine and propofol were determined using reversed-phase high performance liquid chromatography (Yeganeh and Ramzan, 1997; Ji et al., 2004).

\section{PET scanning}

A previously described PET procedure for measuring regional cerebral blood flow (rCBF) was used (Kaisti et al., 2002). Scans were obtained with an ECAT EXACT HR + PET scanner (Siemens/CTI) and $\left[{ }^{15} \mathrm{O}\right] \mathrm{H}_{2} \mathrm{O}(300$ $\mathrm{MBq}$ ) was used as the tracer. Tracer was administered as a $15 \mathrm{~s}$ bolus infusion followed by a $90 \mathrm{~s}$ static List Mode activity acquisition in 3D 
Table 1. Calculated (with STANPUMP software) and measured drug concentrations in plasma during the neuroimaging sessions

\begin{tabular}{|c|c|c|c|c|c|c|c|c|}
\hline \multirow[b]{2}{*}{ Drug } & \multicolumn{2}{|l|}{ Sed } & \multicolumn{2}{|l|}{$\mathrm{LOC}$} & \multicolumn{2}{|l|}{$\mathrm{ROC}$} & \multicolumn{2}{|l|}{ LOC-2 } \\
\hline & Calculated & Measured & Calculated & Measured & Calculated & Measured & Calculated & Measured \\
\hline Dexmedetomidine (ng/ml) & $1.7 \pm 0.4$ & $2.3 \pm 0.7$ & $2.1 \pm 0.6$ & $3.2 \pm 1.3$ & $2.1 \pm 0.6$ & $3.0 \pm 1.1$ & $2.1 \pm 0.7$ & $2.8 \pm 1.1$ \\
\hline Propofol $(\mu \mathrm{g} / \mathrm{ml})$ & $1.6 \pm 0.5$ & $1.3 \pm 0.5$ & $2.2 \pm 0.6$ & $1.8 \pm 0.6$ & $1.6 \pm 0.5$ & $0.6 \pm 0.4$ & NA & NA \\
\hline
\end{tabular}

Sed, sedation; LOC, loss of consciousness; ROC, return of consciousness; $L O C-2$, second loss of consciousness (dexmedetomidine only); $N A=$ not applicable. Data as mean \pm SD.
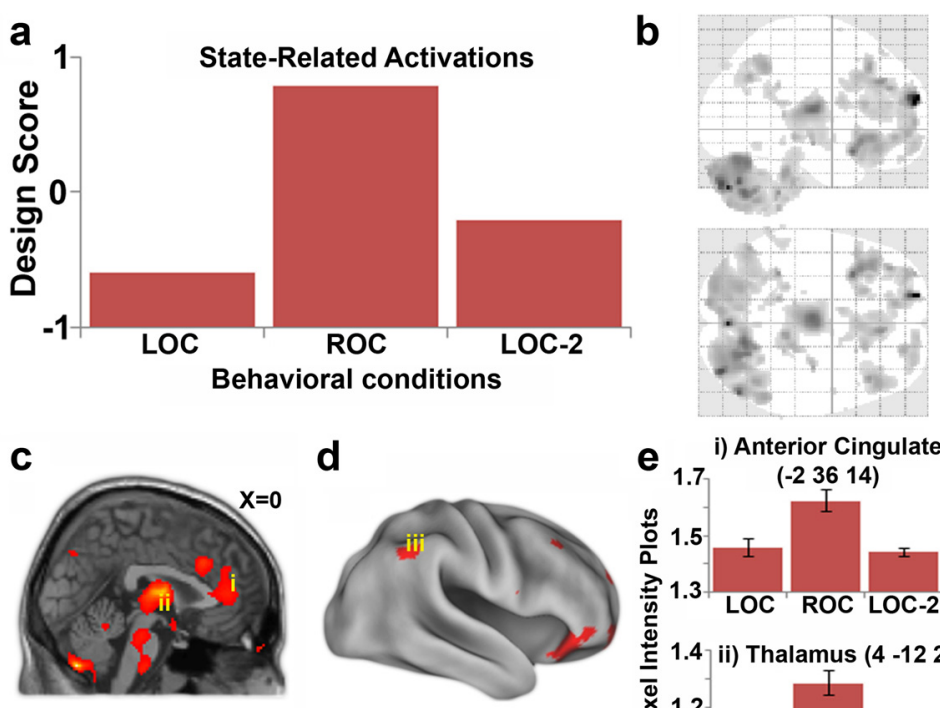

d
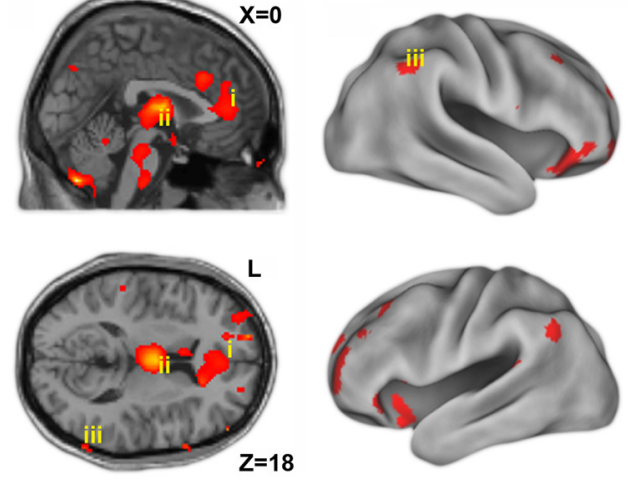

Figure 2. Neural correlates associated with the ROC during constant dose dexmedetomidine anesthesia. $\boldsymbol{a}$, LV1 design score pattern showing changes associated with the temporary return of consciousness $(p<0.0001) . \boldsymbol{b}, \mathrm{PLS}$ singular image (positive salience; top, sagittal; bottom, axial) of LV1 ( $p<0.001)$ projected on SPM-8 glass brain template. c, Sagittal (top) and axial (bottom) sections showing cingulate (i), thalamus (ii), inferior parietal cortex (iii), and brainstem activations. $\boldsymbol{d}$, Cortical renderings showing parietal (iii) and frontal activations. $e$, Voxel intensity plots (mean \pm SE) showing how i, ii, and iii follow LV1 pattern.

mode. Arterial blood activity was measured using a peristaltic roller pump and BGO crystal detector system (Allogg) cross-calibrated with the scanner. All $\left[{ }^{15} \mathrm{O}\right] \mathrm{H}_{2} \mathrm{O}$ scans were corrected for detector dead time, tracer decay, and measured tissue photon attenuation, and reconstructed into a $128 \times 128$ matrix using a 3D transaxial Hann filter with a $4.6 \mathrm{~mm}$ cutoff. The field-of-view was $30 \mathrm{~cm}$, resulting in a pixel size of $300 \mathrm{~mm} /$ $128=2.34 \mathrm{~mm}$. Photon attenuation in the tissue was taken into account according to transmission scans obtained using three external circulating ${ }^{68} \mathrm{Ge}$ rod sources. Individual 1.5 T MRIs were acquired for neuroanatomy (Gyroscan Intera CV Nova Dual; Philips Medical Systems).

\section{Parametric images and preprocessing}

$\left[{ }^{15} \mathrm{O}\right] \mathrm{H}_{2} \mathrm{O}$ images were computed into quantitative parametric $\mathrm{rCBF}$ images using arterial blood tracer activity data and tracer kinetic modeling (Kaisti et al., 2002). Images were then realigned, coregistered, and spatially normalized to Montreal Neurological Institute space using the processing routines implemented within the Statistical Parametric Mapping (SPM) software package (SPM-8; Wellcome Department of Cognitive Neurology, University College London, London, England; available at www.fil.ion.ucl.ac.uk/spm) running under MATLAB 2009b (MathWorks). All normalized PET images were smoothed using an isotropic Gaussian kernel of $12 \mathrm{~mm}$ full-width at half-maximum.

\section{Data analyses}

Images were analyzed for overall patterns of condition-related rCBF changes using partial least-squares (PLS) software (PLS-software; http://www.rotman- drug-specific) effects on consciousness $(n=17)$.

baycrest.on.ca/index.php?section $=84$ ). PLS is a multivariate analysis technique used to detect optimal covariation between brain voxelvalues and the experimental design (Lobaugh et al., 2001; Lin et al., 2003). A mathematical description of the method has been given in detail previously (McIntosh et al., 1996; McIntosh and Lobaugh, 2004).

Analyses were directed at four primary issues. First, to identify brain areas that changed their function with the changing state of consciousness, independent of drug effects, we analyzed the three scans (LOC, ROC, and LOC-2) obtained from the dexmedetomidine group ( $n=8$, due to one missing and one damaged scan). Second, as some suggest that the anesthesia-induced change in consciousness is related to changing functional connectivity within the frontoparietal network (Imas et al., 2005; Alkire et al., 2008; Boveroux et al., 2010; Boly et al., 2011; Ku et al., 2011), we assessed the changes in parietal functional connectivity between the ROC and the LOC-2 scans of the dexmedetomidine group using seed PLS (McIntosh et al., 2003). These particular scans were the least influenced by changes in drug levels or extemporaneous stimulation effects. Third, to understand the generalizability of the results across agents, the four scans (BL, Sed, LOC, ROC) obtained from the propofol group were analyzed for propofol-induced effects on consciousness ( $n=9$, due to a missing scan). Fourth, a combined analysis of the propofol and the dexmedetomidine groups was performed to reveal the condition-related (rather than

\section{Statistical methods}

The statistical significance of each latent variable (LV) arising from the various PLS analyses was determined using permutation tests (McIntosh et al., 1996). In the present study, 1000 permutations were computed. The reliability of the identified saliences was assessed by a bootstrap estimation of the standard error (SE; 1000 iterations), which provides evidence of the stability of the findings rather than simply demonstrates whether an effect exists (McIntosh and Lobaugh, 2004). Voxels with saliences $>3.3$ times their SE (corresponding to an approximate $p<$ 0.001 on a two-tailed normal distribution) were considered reliable. As saliences are identified in one single analytic step, correcting for multiple-comparisons is not necessary in PLS. For clarity, only positive saliences are presented in the figures. The SPM- 8 glass brain template was used in 3D visualization of the PLS analysis results. However, PLS and not SPM was used to generate these findings.

\section{Results}

No adverse events or clinically significant changes were observed in the vital parameters (data not shown). Condition-related drug blood levels are shown in Table 1. 
Table 2. The neural correlates of the return of consciousness during constant level dexmedetomidine revealed by PLS

\begin{tabular}{|c|c|c|c|c|c|}
\hline Brain regions & Brodmann area & MNI coordinates (in mm) $x, y, z$ & Cluster size (voxels) & BSR & $p$ value \\
\hline \multicolumn{6}{|l|}{ Latent Variable 1} \\
\hline \multicolumn{6}{|c|}{ Positive salience—shown in Figure 2} \\
\hline L superior frontal gyrus & 8/9/10/11 & $-20,62,20$ & 793 & 17.26 & $<0.0001$ \\
\hline R anterior prefrontal & $10 / 11$ & $36,62,-24$ & 834 & 16.0008 & $<0.0001$ \\
\hline Cerebellum & & $0,-78,-42$ & 8102 & 15.2835 & $<0.0001$ \\
\hline Superior frontal gyrus & 8/9/10/11 & $14,70,26$ & 231 & 13.6909 & $<0.0001$ \\
\hline Thalamus & $10 / 24$ & $-4,-14,14$ & 1429 & 10.2229 & $<0.0001$ \\
\hline R superior temporal sulcus & 39 & $68,-68,22$ & 219 & 10.0856 & $<0.0001$ \\
\hline L inferior frontal gyrus & $13 / 46 / 47$ & $-36,16,-16$ & 850 & 10.0765 & $<0.0001$ \\
\hline R inferior parietal & $39 / 40$ & $56,-68,50$ & 538 & 8.8778 & $<0.0001$ \\
\hline R medial frontal gyrus & $8 / 9$ & $6,36,34$ & 1204 & 7.9879 & $<0.0001$ \\
\hline L superior temporal gyrus & 38 & $-36,14,-66$ & 112 & 7.4662 & $<0.0001$ \\
\hline R middle frontal gyrus & $6 / 8 / 9$ & $40,28,44$ & 121 & 7.2273 & $<0.0001$ \\
\hline Brainstem/pons & & $8,-36,-32$ & 836 & 7.1674 & $<0.0001$ \\
\hline$R$ middle frontal gyrus & $6 / 8 / 9$ & $32,62,24$ & 155 & 6.7936 & $<0.0001$ \\
\hline R inferior frontal gyrus & $13 / 46 / 47$ & $44,34,-14$ & 645 & 6.611 & $<0.0001$ \\
\hline L inferior parietal & $39 / 40$ & $-54,-62,38$ & 183 & 6.5215 & $<0.0001$ \\
\hline L orbital gyrus & 11 & $-14,56,-56$ & 890 & 6.5092 & $<0.0001$ \\
\hline Anterior cingulate & $24 / 32$ & $-14,48,-6$ & 239 & 6.2658 & $<0.0001$ \\
\hline R orbital gyrus & 11 & $16,58,-66$ & 219 & 5.8429 & $<0.0001$ \\
\hline $\mathrm{L}$ inferior frontal gyrus & $13 / 46 / 47$ & $-30,38,-32$ & 115 & 5.6669 & $<0.0001$ \\
\hline L supramarginal gyrus & 40 & $-72,-52,28$ & 115 & 5.6521 & $<0.0001$ \\
\hline \multicolumn{6}{|l|}{ Negative salience } \\
\hline L parahippocampal gyrus & $20 / 35 / 36$ & $-30,-36,-16$ & 6488 & -22.0599 & $<0.0001$ \\
\hline R lingual gyrus & $17 / 19$ & $18,-44,-6$ & 9395 & -18.9551 & $<0.0001$ \\
\hline R angular gyrus & 39 & $34,-62,36$ & 2176 & -18.1224 & $<0.0001$ \\
\hline L inferior parietal & $39 / 40$ & $-36,-42,42$ & 2122 & -14.0804 & $<0.0001$ \\
\hline R middle frontal gyrus & $6 / 8 / 9$ & $46,4,40$ & 1674 & -13.5639 & $<0.0001$ \\
\hline L postcentral gyrus & $2 / 3 / 4$ & $-12,-40,68$ & 1306 & -6.3560 & $<0.0001$ \\
\hline L lingual gyrus & $17 / 19$ & $-12,-92,-2$ & 143 & -4.3952 & $<0.0001$ \\
\hline
\end{tabular}

Corresponding clusters are shown in Figure 2. MNI, Montreal Neurological Institute; BSR, Bootstrap ratio.

Imaging the ROC during constant level dexmedetomidine

All subjects in the dexmedetomidine group were successfully aroused from unconsciousness into the conscious state with gentle tactile and/or loud verbal stimulation without any alteration in the dosing of the drug. All but one of the subjects subsequently returned to an unconscious state after they were left unstimulated for a few minutes. Multivariate pattern analysis of the PET scans obtained in the three conditions (LOC, ROC, and LOC-2) on dexmedetomidine resulted in three LVs with only LV1 being significant $(p<0.0001)$ and accounting for $80 \%$ of the cross-block covariance between the change in the level of consciousness and the brain activity patterns. The pattern of changes identified in LV1 is shown in Figure 2 and Table 2. The presence of a conscious state was specifically related to activation in a number of brain areas including: the anterior cingulate cortex (ACC) in the medial frontal lobe, the midline thalamus, hypothalamus, the locus ceruleus/parabrachial area in the brainstem, cerebellum, and portions of the lateral orbital frontal and parietal lobes (Fig. $2 b-d$ ). The interpretation of this result is aided by the representative voxel intensity plots provided in Figure $2 e$. These plots show how the identified areas follow the overall pattern of LV1.

\section{Functional connectivity and consciousness}

We further investigated how the change in the state of consciousness and the ability to respond affected the functional connectivity of the parietal cortex when the subjects on dexmedetomidine again changed from the conscious, but sedated condition (ROC), to the second unconscious episode (LOC-2). As presented in a correlation plot difference image in Figure 3 and Table 3, the inferior parietal cortex (Brodmann areas 39/40; Fig. $2 c$, $d$, marker iii) was functionally connected with the rostral ACC and other frontal regions more in the conscious state compared with the unconscious state.

\section{Arousal from propofol-induced unconsciousness}

To understand the generalizability of the findings seen with dexmedetomidine, we analyzed the four sequential PET scans obtained from the subjects who received propofol-TCI in a stepwise increasing manner at the four different levels of consciousness (BL, Sed, LOC, and ROC). PLS analysis of these four conditions yielded four LVs associated with the changing levels of consciousness. The first and the second latent variables (LV1 and LV2) accounted for $68 \%$ (LV1, $p<0.0001)$ and $23 \%$ (LV2, $p<0.03$ ) of the cross-block covariance. The third latent variable (LV3) was not significant and the fourth latent variable (LV4) represented the residuals. The relevant results for the comparison with the dexmedetomidine findings are those found with LV2 (Fig. 4). With propofol, the ROC was associated with the activation of anterior arousal networks of the thalamus, brainstem, hypothalamus, and the dorsal ACC (Fig. $4 b, c, e$ ). Additional activation was observed in the precuneus/posterior cingulate region (Fig. $4 b, c$ ). Compared with the dexmedetomidine results, the emergence from propofol anesthesia showed even less neocortical activation (Fig. 4c,d).

\section{Combined analysis of arousal from unconsciousness}

To reduce drug-specific effects on the results and to further dissociate the brain activity changes related to the different states of consciousness from those more likely to be specifically associated with either dexmedetomidine or propofol, we combined the 
a

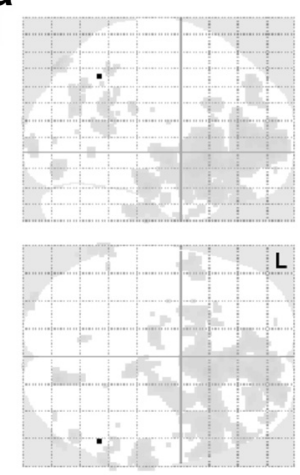

b
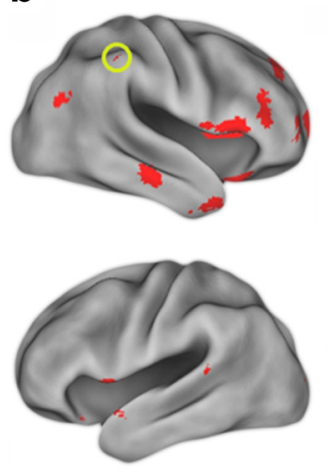

C
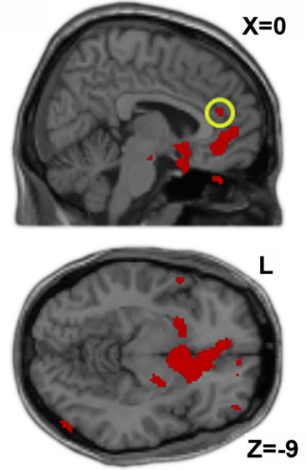

d

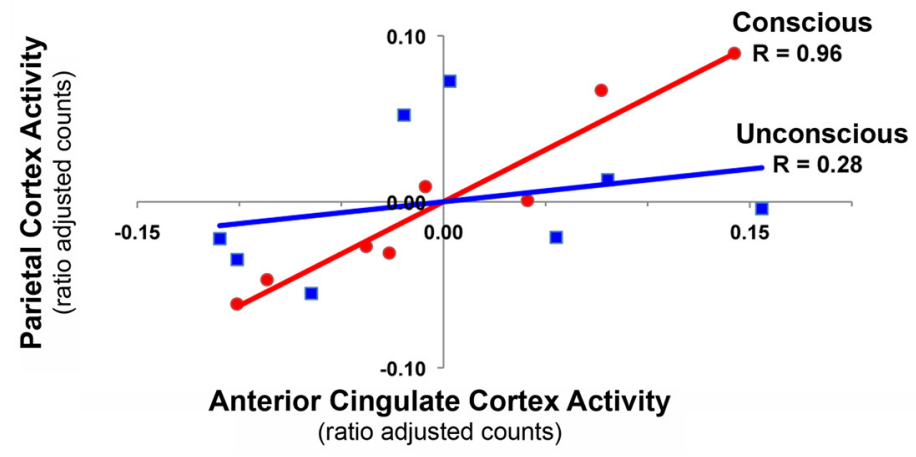

Figure 3. Consciousness-related functional connectivity differences of the right parietal cortex during constant dose dexmedetomidine anesthesia. The parietal region $(x=52, y=-52, z=46)$ is functionally connected with cingulate $(x=-4, y=36$, $z=20$ ) and other ventral forebrain regions when conscious. $\boldsymbol{a}$, Projections (top, sagittal; bottom, axial; PLS analysis results projected on SPM-8 glass brain template) showing regions significantly more correlated with the seed voxel (black dot) during consciousness. $\boldsymbol{b}$, Renderings reveal cortical areas more connected when conscious. c, Sagittal (top) and axial (bottom) sections showing effects in cingulate, hypothalamus, and basal forebrain. $\boldsymbol{d}$, Correlation plot showing state-related functional connectivity changes between parietal (left circle) and cingulate (right circle) activity. Linear regressions, conscious (red); unconscious (blue).

groups for a condition-related PLS analysis. This analysis used the scans from the four states (BL, Sed, LOC, and ROC) and yielded four LVs associated with the changing levels of consciousness. The first and the second latent variables (LV1 and LV2) accounted for 68 and $25 \%$ of the cross-block covariance, respectively ( $p<0.0001$, for both). The third latent variable (LV3) was not significant and the fourth latent variable (LV4) again represented the residuals.

Figure 5 and Table 4 shows the relevant arousal-related LV2 pattern of activity, which was associated with the return of a conscious state following drug-induced unconsciousness. Activation was again seen in the anterior brain arousal networks (i.e., thalamus, hypothalamus, the locus ceruleus/parabrachial area, and the ACC) when unconscious subjects regained their consciousness and became responsive to external stimuli. In this combined analysis, the ROC was not associated with a significant restoration of cortical activation (Fig. $5 d$ ).

\section{Combined analysis of drug-related suppressions associated with anesthesia}

The other activity pattern (LV1) found in the PLS analysis of combined groups (Fig. 6 and Table 4) revealed suppressed regional brain activity in bilateral frontoparietal regions, the precuneus/posterior cingulate, the ACC in the medial prefrontal cortex, the thalamus, and in the brainstem (Fig. $6 b-d$ ). Representative voxels that follow this pattern of activity are shown in Figure $6 e$. The LV1 patterns for each anesthetic separately displayed similar regional findings (data not shown).

\section{Discussion}

Most neuroimaging studies of anesthetic action assume that loss of functional activity reflects the neural correlates of consciousness (Heinke and Schwarzbauer, 2002; Alkire et al., 2008; Brown et al., 2010). Yet, this approach lacks the ability to distinguish the consciousness-related changes from the epiphenomenal, e.g., drug-related, effects. Here, we used a different approach and investigated what turns back on when consciousness reemerges following anesthetic-induced unconsciousness. The structures that activated when consciousness resumed were the brainstem, the thalamus, the hypothalamus, and the ACC (Figs. 2, 4, 5). Although only limited frontal and parietal activity was observed during the ROC, an active parietal region demonstrated greater functional connectivity with the ACC (and other frontal regions) during the conscious state (Fig. 3). These findings reveal a functional network that activates with restored consciousness to enable arousal, the subjective awareness of stimuli, and the behavioral expression of the contents of consciousness (Fig. 1).

Arousal-induced activations were mostly localized in deep, phylogenetically old brain structures rather than in the neocortex. These regions reactivated upon returning consciousness in subjects receiving a constant dexmedetomidine infusion

(Fig. 2) but also in subjects recovering from propofol-induced unconsciousness (Fig. 4), suggesting that these deep brain structures form a common foundation for a conscious state. These structures activate also upon awakening from natural Stage 2 sleep (Balkin et al., 2002), and their direct stimulation can reverse the unconsciousness of anesthesia (Alkire et al., 2007) or improve behavioral arousal following chronic brain injury (Schiff et al., 2007). On the other hand, impaired consciousness or coma is known to result from paramedian thalamic and midbrain infarcts (Castaigne et al., 1981).

The arousal findings (LV2) associated with propofol (Fig. 4) were consistent with the dexmedetomidine state-related findings (Fig. 2). The separate analyses for both anesthetic agents identified brainstem, thalamus, hypothalamus, and ACC as regions reactivating upon returning consciousness. With propofol, the concentration of the drug was decreasing as subjects regained consciousness, suggesting the regional findings might be attributable to a changing drug level. However, as there was no change in drug dosing with dexmedetomidine and there was strong similarity of findings between dexmedetomidine and propofol, it seems likely that the observed changes were due to an altered state of consciousness and not to any particular interaction with drug dose. The only difference in the regional findings between these drugs was the activation of precuneus/posterior cingulate region during the recovery from propofol anesthesia, which could suggest a propofol-specific effect on this brain region.

Xie et al. (2011) also examined the gain of function associated with the restoration of consciousness during constant dose anes- 
Table 3. Right inferior parietal cortex functional connectivity changes associated with the changing state of consciousness during constant level dexmedetomidine revealed by PLS

\begin{tabular}{|c|c|c|c|c|c|}
\hline Brain regions & Brodmann area & MNI coordinates (in mm) $x, y, z$ & Cluster size (voxels) & BSR & $p$ value \\
\hline \multicolumn{6}{|l|}{ Positive correlations - shown in Figure 3} \\
\hline $\mathrm{R}$ precuneus/superior posterior parietal & 7 & $14,-80,56$ & 220 & 4.3339 & $<0.0001$ \\
\hline $\mathrm{R}$ anterior cingulate/medial frontal/caudate & $10 / 24 / 32$ & $10,18,-4$ & 3326 & 4.2181 & $<0.0001$ \\
\hline R inferior frontal gyrus & $13 / 46 / 47$ & $52,40,12$ & 207 & 3.5084 & 0.0005 \\
\hline R inferior temporal gyrus & 20 & $66,-22,-22$ & 266 & 3.4935 & 0.0005 \\
\hline R inferior frontal gyrus & $13 / 46 / 47$ & $24,16,-22$ & 333 & 3.4 & 0.0007 \\
\hline R middle temporal gyrus & $21 / 37 / 39$ & $46,12,-40$ & 106 & 3.3091 & 0.0009 \\
\hline R inferior occipital gyrus & & $52,-80,-12$ & 106 & 3.2705 & 0.0011 \\
\hline Pons & & $10,-32,-36$ & 124 & 3.1521 & 0.0016 \\
\hline Insula & 13 & $42,20,10$ & 667 & 3.1009 & 0.0019 \\
\hline R inferior parietal & $39 / 40$ & $58,-46,52$ & 125 & 3.0594 & 0.0022 \\
\hline R superior frontal gyrus & $8 / 9 / 10 / 11$ & $28,44,46$ & 189 & 2.816 & 0.0049 \\
\hline R supramarginal gyrus & 40 & $66,-48,28$ & 126 & 2.7575 & 0.0058 \\
\hline Putamen & & $-28,14,2$ & 197 & 2.7574 & 0.0058 \\
\hline \multicolumn{6}{|l|}{ Negative correlations } \\
\hline R middle frontal gyrus & $6 / 8 / 9$ & $22,-12,64$ & 5092 & -6.0758 & $<0.0001$ \\
\hline L postcentral gyrus & $2 / 3 / 4$ & $-52,-18,54$ & 1325 & -4.5581 & $<0.0001$ \\
\hline R lingual gyrus & $17 / 19$ & $22,-104,-14$ & 210 & -3.969 & 0.0001 \\
\hline Rprecuneus & 7 & $18,-52,62$ & 173 & -3.3259 & 0.0009 \\
\hline
\end{tabular}

Corresponding clusters are shown in Figure 3.

thesia. By using physostigmine, a centrally acting anticholinesterase drug, they were able to increase brain acetylcholine levels and reverse propofol-induced unconsciousness. In subjects who responded, thalamus, lateral parietal cortex, and precuneus reactivated with restored consciousness. Thus, in their study and in the present study, a conscious state was associated with the activation of the thalamus and the parietal cortex. In our study, however, only the subjects anesthetized with propofol showed precuneal activation upon arousal, further suggesting that the activation of precuneus might be a propofolspecific finding. Another difference between these studies is that Xie et al.(2011) did not find activation of the ACC. As consciousness was restored in the dexmedetomidine subjects without any pharmacological manipulation, it seems possible that the physostigmine used by Xie et al. (2011) could have influenced their regional findings.

Perhaps unexpectedly, the return to a conscious state was not accompanied by large changes in neocortical function. This lack of a cortical response was especially apparent with propofol (Fig. 4d) and in the combined analysis of the propofol and dexmedetomidine scans (Fig. 5d). This phenomenon may be more understandable from a clinical perspective. The recovery from anesthesia does not occur all at once, but rather it appears to occur in a bottom-up manner. When emerging from deep anesthesia there will first be signs of autonomic arousal, followed by a slow return of brainstem reflexes, eventually leading to reflexive or uncoordinated somatic movements that occur somewhat before subjects can willfully respond to simple commands. As shown in our results, only minimal cortical activity is neces-
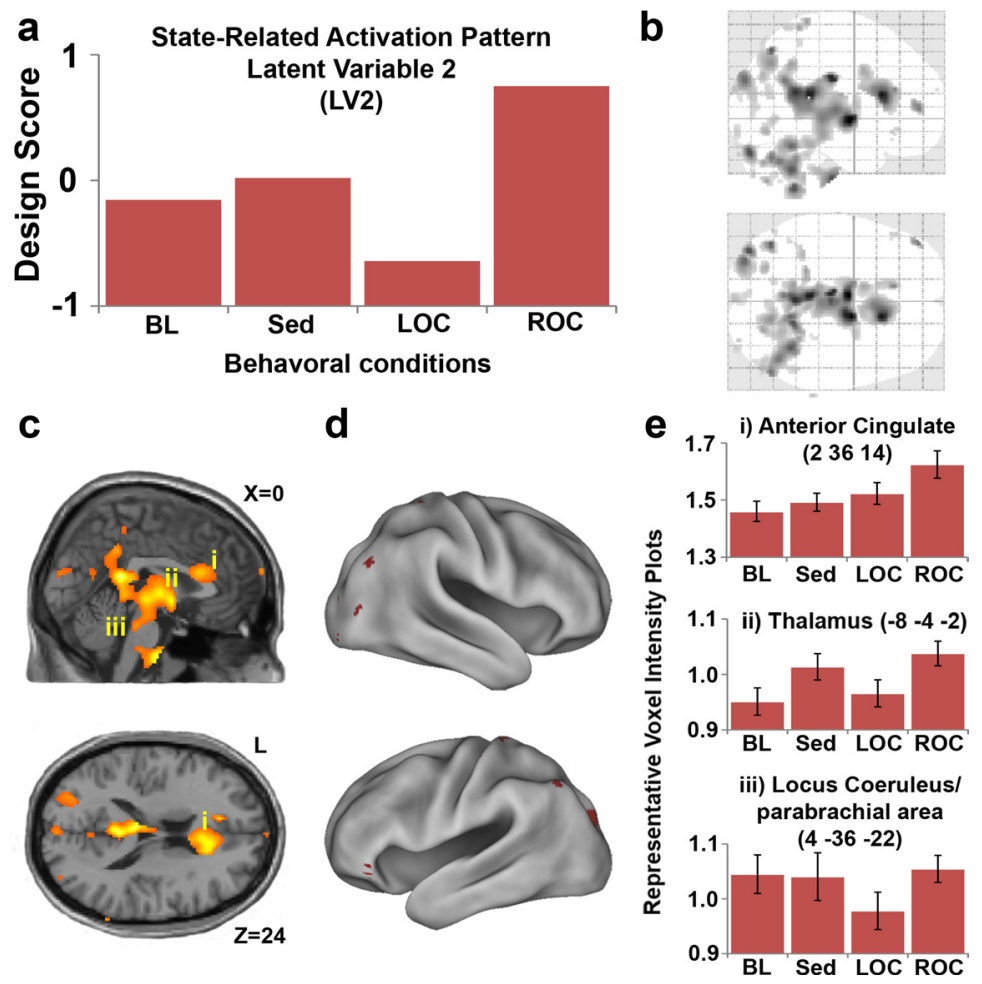

Figure 4. Neural correlates associated with the return of consciousness following propofol anesthesia. $\boldsymbol{a}$, LV2 design score pattern shows changes primarily associating with $\operatorname{ROC}(p<0.03) . \boldsymbol{b}$, PLS singular image (positive salience; top, sagittal; bottom, axial) showing the regions associated with LV2 $(p<0.001)$ projected on SPM-8 glass brain template. $c$, Sagittal (top) and axial (bottom) sections showing activation in the ACC (i), thalamus (ii), and the brainstem (iii). $\boldsymbol{d}$, Cortical renderings showing minimal occipital, parietal, and frontal activations at this threshold. $e$, Region-specific voxel intensity plots showing how i, ii, and iii follow the overall pattern of LV2 (mean $\pm \mathrm{SE}$ ).

sary at this point. Thus, emergence of a conscious state, the essential foundation of consciousness (Fig. 1), precedes the full recovery of neocortical processing required for rich conscious experiences. We propose that the failure of simple processed EEG monitoring technology to detect patient awareness during anesthesia is at least partly due to the aforementioned pattern of brain arousal (Avidan et al., 2008, 2011; Kaskinoro et al., 2011). 

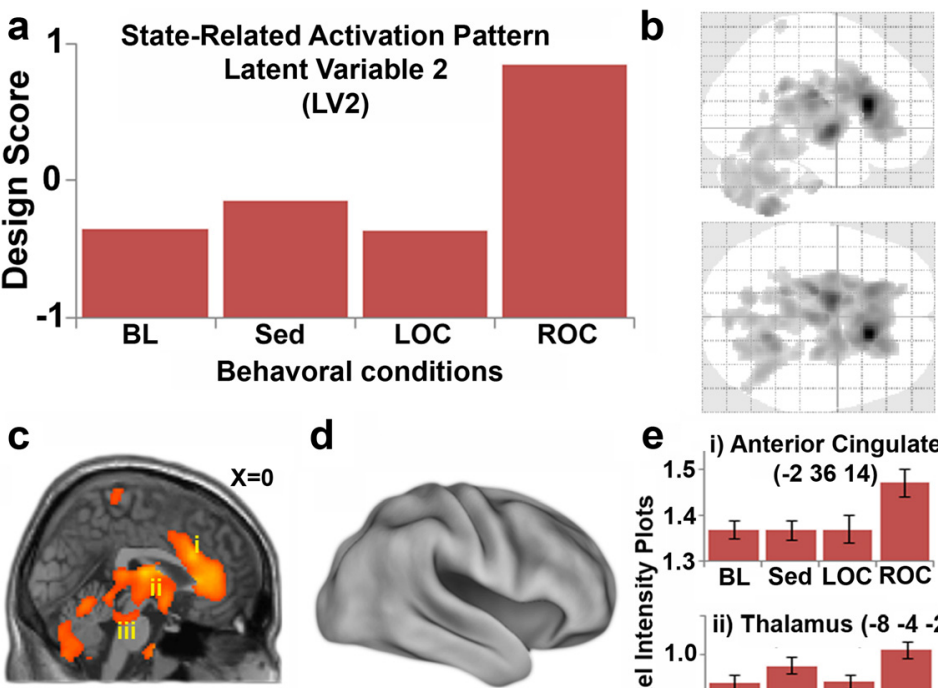

d
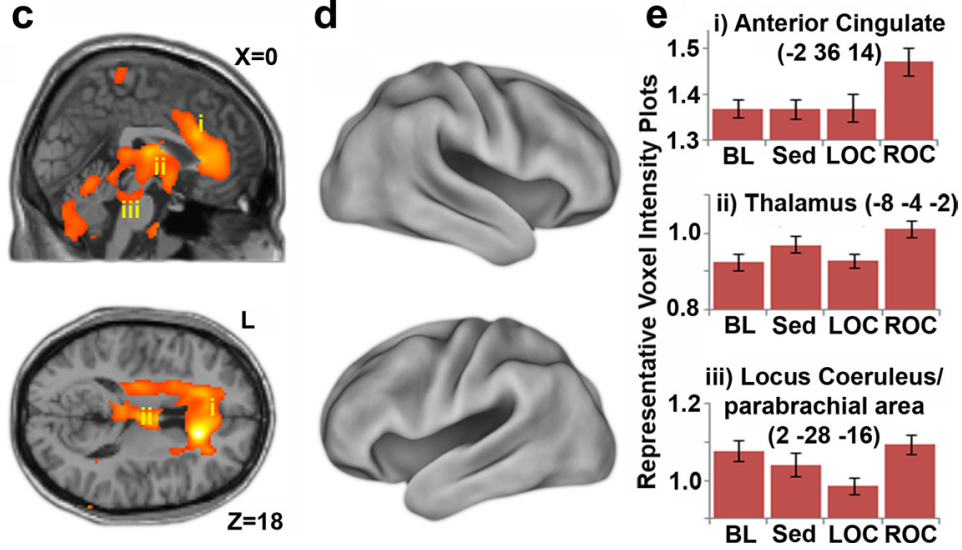

Figure 5. Neural correlates associated with the return of consciousness (groups combined). $\boldsymbol{a}, \mathrm{LV} 2$ design score pattern shows changes primarily associated with $\operatorname{ROC}(p<0.0001) \cdot \boldsymbol{b}$, PLS singular image (positive salience; top, sagittal; bottom, axial) showing regions associated with LV2 $(p<0.001)$ projected on SPM-8 glass brain template. c, Sagittal (top) and axial (bottom) sections showing activation in the ACC (i), thalamus (ii), and the brainstem (iii) locus ceruleus/parabrachial area. $\boldsymbol{d}$, Cortical renderings showing no activations at this threshold.e, Region-specific voxel intensity plots showing how i, ii, and iii follow the overall pattern of LV2 (mean $\pm \mathrm{SE}$ ). Abbreviations as in Figure 4.

All of these data are in agreement with the experiences obtained from hydranencephalic children, who are devoid of nearly the entire cerebral cortex and yet still display conscious-like behavior (Merker, 2007). Although these children have clear deficits in experiencing the rich contents of consciousness, they undoubtedly are in a conscious state, supporting the idea that the subcortical areas identified in our study play a fundamental role in consciousness. Indeed, numerous studies indicate that consciousness as a process involving both the conscious state (Schiff and Plum, 2000; Lydic and Baghdoyan, 2005) and the contents of consciousness (Baars et al., 2003) likely arises from the interactions between cortical and subcortical mechanisms working through specific network connectivity within the brain (Baars, 1995; Tononi, 2004; Xie et al., 2011).

A number of studies have now examined the connectivity effects associated with changing levels of consciousness (Imas et al., 2005; Massimini et al., 2005; Boveroux et al., 2010; Mhuircheartaigh et al., 2010; Stamatakis et al., 2010; Boly, 2011; Boly et al., 2011; Schrouff et al., 2011). It has been hypothesized that decreased frontal to parietal feedback is a likely correlate for the diminished consciousness associated with sleep (Massimini et al., 2005), brain injury (Boly et al., 2011), and anesthesia (Imas et al., 2005; Alkire et al., 2008; Ku et al., 2011). Our functional connectivity findings with dexmedetomidine (Fig. 3) revealed that the inferior parietal area correlated with frontal activity in the conscious state, but not so in the unconscious state. This demonstrates that functional connectivity exists between frontal and parietal brain areas when the subjects are conscious, but that connectivity fades with LOC. This is not a drug-dose driven change in functional connectivity because the drug concentra- tion remained constant. Our findings are thus consistent with the emerging idea that frontoparietal connectivity plays some role in conscious information processing (Alkire et al., 2008; Ku et al., 2011).

Suppressed frontoparietal and thalamic activity appear to be a common finding in most anesthesia studies (Kaisti et al., 2002; Baars et al., 2003; Bonhomme et al., 2008; Mhuircheartaigh et al., 2010; Stamatakis et al., 2010; Schrouff et al., 2011; Tu et al., 2011). These changes have generally been interpreted directly as neural correlates for LOC. However, since these anesthetic-induced regional deactivations could simply represent the brain areas most affected by the drug used to induce unconsciousness, establishing a causal relationship between these changes and consciousness is difficult. Indeed, pattern analysis across the four states of consciousness revealed that these brain regions do follow the overall pattern of drug level changes (Fig. 6), and should thus be interpreted with caution.

One distinct cortical region activating during the recovery of consciousness was the ACC (Figs. 2-5). This medial prefrontal region has been proposed to play a critical role in consciousness by integrating cognitive-emotional processing with the state of arousal and the intent-to-act (Paus, 2001). Others suggest that the ACC is a key site of self-regulation of behavior (Posner et al., 2007), or might even be involved with the feeling of "free will" (Crick, 1994). The neuroanatomy of the ACC and its connections with motor control regions suggest that this brain structure acts as the neural interface for translating intentions into actions. This idea is in agreement with studies showing that restored activity in the ACC correlates with the level of responsiveness in brain-injured patients (Qin et al., 2010; Zhou et al., 2011). Our inferior parietal finding may have importance because of its functional connection with the ACC during the conscious state and because of its suggested role in movement intention and motor awareness (Desmurget and Sirigu, 2009). In work presented by Desmurget et al. (2009), direct electrical stimulation of the inferior parietal cortex (Brodmann areas 39/40) produced in awake brain surgery patients the sensation of a "will to move." Desmurget and colleagues (2009) concluded that conscious intention and motor awareness arise from increased parietal activity before movement execution. This idea fits well with our findings.

In summary, we studied the emergence of consciousness, as manifested by the return of its behavioral expression. We have shown that ROC after a period of anesthetic-induced unconsciousness involves activation of the brainstem, thalamus, and the ACC arousal networks as well as restored functional connectivity within the frontoparietal network.

\section{References}

Alkire MT, McReynolds JR, Hahn EL, Trivedi AN (2007) Thalamic microinjection of nicotine reverses sevoflurane-induced loss of righting reflex in the rat. Anesthesiology 107:264-272.

Alkire MT, Hudetz AG, Tononi G (2008) Consciousness and anesthesia. Science 322:876-880. 
Table 4. The regional changes associated with the return of consciousness and the loss of consciousness observed in the combined analysis of dexmedetomidine and propofol revealed by PLS

\begin{tabular}{|c|c|c|c|c|c|}
\hline Brain regions & Brodmann area & MNI coordinates (in mm) $x, y, z$ & Cluster size (voxels) & BSR & $p$ value \\
\hline \multicolumn{6}{|l|}{ Latent variable 2} \\
\hline \multicolumn{6}{|l|}{ Positive salience—-shown in Figure 5} \\
\hline Anterior cingulate/caudate/thalamus & $10 / 24$ & $12,24,16$ & 10341 & 10.5547 & $<0.0001$ \\
\hline$R$ cerebellum & & $16,-52,-58$ & 2694 & 6.0852 & $<0.0001$ \\
\hline L cerebellum & & $-12,-64,-46$ & 669 & 5.0053 & $<0.0001$ \\
\hline Pons & & $2,-12,-40$ & 167 & 4.6936 & $<0.0001$ \\
\hline Thalamus & $10 / 24$ & $26,-32,6$ & 123 & 4.0715 & $<0.0001$ \\
\hline \multicolumn{6}{|l|}{ Negative salience } \\
\hline R parahippocampal gyrus & $20 / 35 / 36$ & $24,-8,-28$ & 5591 & -16.6896 & $<0.0001$ \\
\hline L parahippocampal gyrus & $20 / 35 / 36$ & $-28,-34,-18$ & 6551 & -11.0476 & $<0.0001$ \\
\hline R postcentral gyrus & $2 / 3 / 4$ & $58,-20,24$ & 4541 & -6.4574 & $<0.0001$ \\
\hline R inferior parietal & $39 / 40$ & $42,-46,48$ & 270 & -5.7562 & $<0.0001$ \\
\hline$R$ cuneus & $17 / 18$ & $6,-106,-4$ & 462 & -5.7045 & $<0.0001$ \\
\hline L precentral gyrus & $4 / 6 / 9$ & $-38,2,36$ & 202 & -4.6472 & $<0.0001$ \\
\hline L middle temporal gyrus & $21 / 37 / 39$ & $-58,-62,-6$ & 324 & -4.6033 & $<0.0001$ \\
\hline $\mathrm{L}$ cerebellum/culmen & & $-4,44,-28$ & 123 & -4.1926 & $<0.0001$ \\
\hline \multicolumn{6}{|l|}{ Latent variable 1} \\
\hline \multicolumn{6}{|l|}{ Positive salience— - shown in Figure 6} \\
\hline R inferior parietal/precuneus & $39 / 40 / 7$ & $52,-54,48$ & 9744 & 12.8661 & $<0.0001$ \\
\hline L inferior parietal/angular gyrus & 39 & $-48,-72,36$ & 4560 & 12.5331 & $<0.0001$ \\
\hline R middle temporal gyrus & $21 / 37 / 39$ & $76,-28,-16$ & 920 & 9.552 & $<0.0001$ \\
\hline $\mathrm{R}$ cerebellum & & $32,-78,-50$ & 3353 & 9.4273 & $<0.0001$ \\
\hline L orbital gyrus & $10 / 47$ & $-14,36,-28$ & 19859 & 8.3257 & $<0.0001$ \\
\hline $\mathrm{L}$ cerebellum & & $-36,-78,-46$ & 4329 & 6.8598 & $<0.0001$ \\
\hline Thalamus/medial dorsal nucleus & $10 / 24$ & $8,-14,0$ & 2642 & 6.8006 & $<0.0001$ \\
\hline R superior frontal gyrus & $8 / 9 / 10 / 11$ & $14,36,54$ & 687 & 5.9129 & $<0.0001$ \\
\hline Brainstem & & $4,-24,-50$ & 103 & 5.9054 & $<0.0001$ \\
\hline \multicolumn{6}{|l|}{ Negative salience } \\
\hline R superior temporal gyrus & 38 & $40,-46,14$ & 54536 & -19.4447 & $<0.0001$ \\
\hline
\end{tabular}

Corresponding clusters are shown in Figures 5 and 6.

Avidan MS, Zhang L, Burnside BA, Finkel KJ, Searleman AC, Selvidge JA, Saager L, Turner MS, Rao S, Bottros M, Hantler C, Jacobsohn E, Evers AS (2008) Anesthesia awareness and the bispectral index. N Engl J Med 358:1097-1108.

Avidan MS, Jacobsohn E, Glick D, Burnside BA, Zhang L, Villafranca A, Karl L, Kamal S, Torres B, O'Connor M, Evers AS, Gradwohl S, Lin N, Palanca BJ, Mashour GA (2011) Prevention of intraoperative awareness in a high-risk surgical population. N Engl J Med 365:591-600.

Baars BJ (1995) Tutorial commentary: surprisingly small subcortical structures are needed for the state of waking consciousness, while cortical projection areas seem to provide perceptual contents of consciousness. Conscious Cogn 4:159-162.

Baars BJ, Ramsøy TZ, Laureys S (2003) Brain, conscious experience and the observing self. Trends Neurosci 26:671-675.

Balkin TJ, Braun AR, Wesensten NJ, Jeffries K, Varga M, Baldwin P, Belenky G, Herscovitch $P$ (2002) The process of awakening: a PET study of regional brain activity patterns mediating the re-establishment of alertness and consciousness. Brain 125:2308-2319.

Beecher HK (1947) Anesthesia's second power: probing the mind. Science 105:164-166.

Boly M (2011) Measuring the fading consciousness in the human brain. Curr Opin Neurol 24:394-400.

Boly M, Garrido MI, Gosseries O, Bruno MA, Boveroux P, Schnakers C, Massimini M, Litvak V, Laureys S, Friston K (2011) Preserved

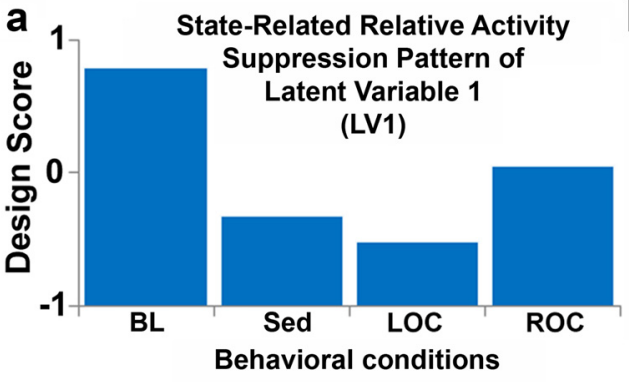

b

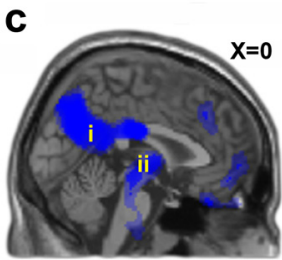

d
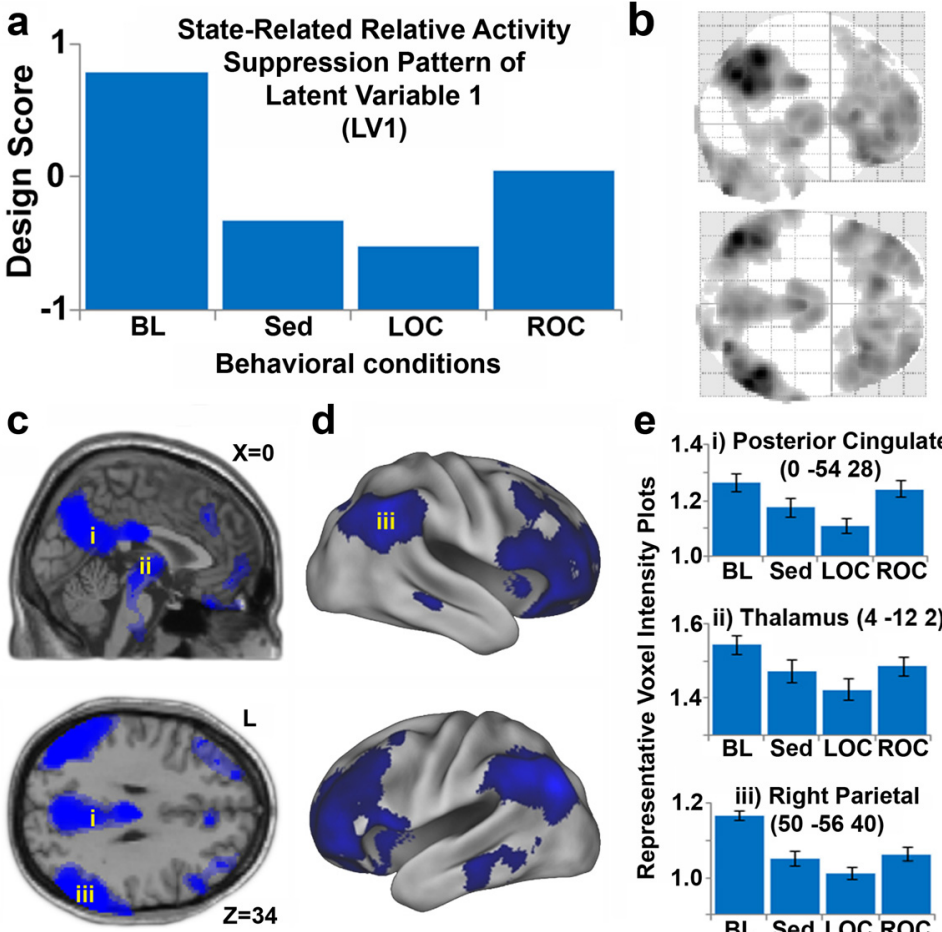

e

1.4 i) Posterior Cingulate

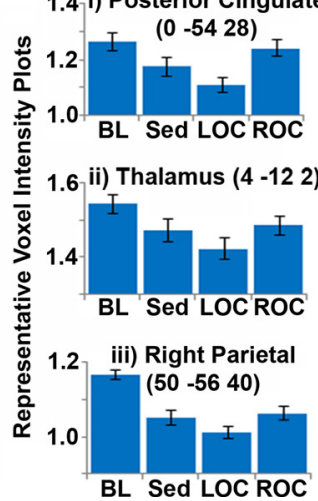

Figure 6. State-related activity suppressions that follow the anesthetic dose (groups combined). $\boldsymbol{a}$, LV1 design score pattern across the four conditions ( $p<0.0001)$. $\boldsymbol{b}$, PLS singular image (positive salience; top, sagittal; bottom, axial) showing regions associated with LV1 ( $p<0.001$ ) projected on SPM-8 glass brain template. c, Sagittal (top) and axial (bottom) sections showing suppression in the posterior cingulate (i), thalamus (ii), inferior parietal (iii), and frontal cortices, precuneus, and brainstem. Light blue, $p<0.001$; dark blue, $p<0.0005$. $\boldsymbol{d}$, Cortical renderings showing frontal, parietal (iii) and temporal suppression. $\boldsymbol{e}$, Voxel intensity plots (mean $\pm \mathrm{SE}$ ) showing how i, ii, and iii follow LV1 pattern. Abbreviations as in Figure 4. 
feedforward but impaired top-down processes in the vegetative state. Science 332:858-862.

Bonhomme V, Maquet P, Phillips C, Plenevaux A, Hans P, Luxen A, Lamy M, Laureys S (2008) The effect of clonidine infusion on distribution of regional cerebral blood flow in volunteers. Anesth Analg 106:899-909.

Boveroux P, Vanhaudenhuyse A, Bruno MA, Noirhomme Q, Lauwick S, Luxen A, Degueldre C, Plenevaux A, Schnakers C, Phillips C, Brichant JF, Bonhomme V, Maquet P, Greicius MD, Laureys S, Boly M (2010) Breakdown of within- and between-network resting state functional magnetic resonance imaging connectivity during propofol-induced loss of consciousness. Anesthesiology 113:1038-1053.

Brown EN, Lydic R, Schiff ND (2010) General anesthesia, sleep, and coma. N Engl J Med 363:2638-2650.

Castaigne P, Lhermitte F, Buge A, Escourolle R, Hauw JJ, Lyon-Caen O (1981) Paramedian thalamic and midbrain infarct: clinical and neuropathological study. Ann Neurol 10:127-148.

Crick, F (1994) The astonishing hypothesis. New York; Scribner.

Crick F, Koch C (2003) A framework for consciousness. Nat Neurosci 6:119-126.

Dehaene S, Changeux JP (2011) Experimental and theoretical approaches to conscious processing. Neuron 70:200-227.

Desmurget M, Sirigu A (2009) A parietal-premotor network for movement intention and motor awareness. Trends Cogn Sci 13:411-419.

Desmurget M, Reilly KT, Richard N, Szathmari A, Mottolese C, Sirigu A (2009) Movement intention after parietal cortex stimulation in humans. Science 324:811-813.

Franks NP (2008) General anaesthesia: from molecular targets to neuronal pathways of sleep and arousal. Nat Rev Neurosci 9:370-386.

Friedman EB, Sun Y, Moore JT, Hung HT, Meng QC, Perera P, Joiner WJ, Thomas SA, Eckenhoff RG, Sehgal A, Kelz MB (2010) A conserved behavioral state barrier impedes transitions between anesthetic-induced unconsciousness and wakefulness: evidence for neural inertia. PLoS One 5:e11903.

Fuller PM, Sherman D, Pedersen NP, Saper CB, Lu J (2011) Reassessment of the structural basis of the ascending arousal system. J Comp Neurol 519:933-956.

Heinke W, Schwarzbauer C (2002) In vivo imaging of anaesthetic action in humans: approaches with positron emission tomography (PET) and functional magnetic resonance imaging (fMRI). Br J Anaesth 89:112-122.

Imas OA, Ropella KM, Ward BD, Wood JD, Hudetz AG (2005) Volatile anesthetics disrupt frontal-posterior recurrent information transfer at gamma frequencies in rat. Neurosci Lett 387:145-150.

Ji QC, Zhou JY, Gonzales RJ, Gage EM, El-Shourbagy TA (2004) Simultaneous quantitation of dexmedetomidine and glucuronide metabolites (G-Dex-1 and G-Dex-2) in human plasma utilizing liquid chromatography with tandem mass spectrometric detection. Rapid Commun Mass Spectrom 18:1753-1760.

Kaisti KK, Metsähonkala L, Teräs M, Oikonen V, Aalto S, Jääskeläinen S, Hinkka S, Scheinin H (2002) Effects of surgical levels of propofol and sevoflurane anesthesia on cerebral blood flow in healthy subjects studied with positron emission tomography. Anesthesiology 96:1358-1370.

Kaskinoro K, Maksimow A, Långsjö J, Aantaa R, Jääskeläinen S, Kaisti K, Särkelä M, Scheinin H (2011) Wide inter-individual variability of bispectral index and spectral entropy at loss of consciousness during increasing concentrations of dexmedetomidine, propofol, and sevoflurane. Br J Anaesth 107:573-580.

Ku SW, Lee U, Noh GJ, Jun IG, Mashour GA (2011) Preferential inhibition of frontal-to-parietal feedback connectivity is a neurophysiologic correlate of general anesthesia in surgical patients. PLoS One 6:e25155.

Lamme VA (2006) Toward a true neural stance on consciousness. Trends Cogn Sci 10:494-501.

Lin FH, McIntosh AR, Agnew JA, Eden GF, Zeffiro TA, Belliveau JW (2003) Multivariate analysis of neuronal interactions in the generalized partial least squares framework: simulations and empirical studies. Neuroimage 20:625-642.

Lobaugh NJ, West R, McIntosh AR (2001) Spatiotemporal analysis of experimental differences in event-related potential data with partial least squares. Psychophysiology 38:517-530.
Lydic R, Baghdoyan HA (2005) Sleep, anesthesiology, and the neurobiology of arousal state control. Anesthesiology 103:1268-1295.

Maksimow A, Snapir A, Särkelä M, Kentala E, Koskenvuo J, Posti J, Jääskeläinen SK, Hinkka-Yli-Salomäki S, Scheinin M, Scheinin H (2007) Assessing the depth of dexmedetomidine-induced sedation with electroencephalogram (EEG)-based spectral entropy. Acta Anaesthesiol Scand 51:22-30.

Marsh B, White M, Morton N, Kenny GN (1991) Pharmacokinetic model driven infusion of propofol in children. Br J Anaesth 67:41-48.

Massimini M, Ferrarelli F, Huber R, Esser SK, Singh H, Tononi G (2005) Breakdown of cortical effective connectivity during sleep. Science 309:2228-2232.

McIntosh AR, Lobaugh NJ (2004) Partial least squares analysis of neuroimaging data: applications and advances. Neuroimage 23 [Suppl] 1:S250-S263.

McIntosh AR, Bookstein FL, Haxby JV, Grady CL (1996) Spatial pattern analysis of functional brain images using partial least squares. Neuroimage 3:143-157.

McIntosh AR, Rajah MN, Lobaugh NJ (2003) Functional connectivity of the medial temporal lobe relates to learning and awareness. J Neurosci 23:6520-6528.

Merker B (2007) Consciousness without a cerebral cortex: a challenge for neuroscience and medicine. Behav Brain Sci 30:63-81; discussion 81-134.

Mhuircheartaigh RN, Rosenorn-Lanng D, Wise R, Jbabdi S, Rogers R, Tracey I (2010) Cortical and subcortical connectivity changes during decreasing levels of consciousness in humans: a functional magnetic resonance imaging study using propofol. J Neurosci 30:9095-9102.

Paus T (2001) Primate anterior cingulate cortex: where motor control, drive and cognition interface. Nat Rev Neurosci 2:417-424.

Posner MI, Rothbart MK, Sheese BE, Tang Y (2007) The anterior cingulate gyrus and the mechanism of self-regulation. Cogn Affect Behav Neurosci 7:391-395.

Qin P, Di H, Liu Y, Yu S, Gong Q, Duncan N, Weng X, Laureys S, Northoff G (2010) Anterior cingulate activity and the self in disorders of consciousness. Hum Brain Mapp 31:1993-2002.

Schiff ND, Plum F (2000) The role of arousal and "gating" systems in the neurology of impaired consciousness. J Clin Neurophysiol 17:438-452.

Schiff ND, Giacino JT, Kalmar K, Victor JD, Baker K, Gerber M, Fritz B, Eisenberg B, Biondi T, O'Connor J, Kobylarz EJ, Farris S, Machado A, McCagg C, Plum F, Fins JJ, Rezai AR (2007) Behavioural improvements with thalamic stimulation after severe traumatic brain injury. Nature 448:600-603.

Schrouff J, Perlbarg V, Boly M, Marrelec G, Boveroux P, Vanhaudenhuyse A, Bruno MA, Laureys S, Phillips C, Pélégrini-Issac M, Maquet P, Benali H (2011) Brain functional integration decreases during propofol-induced loss of consciousness. Neuroimage 57:198-205.

Stamatakis EA, Adapa RM, Absalom AR, Menon DK (2010) Changes in resting neural connectivity during propofol sedation. PLoS One 5:e14224.

Talke P, Lobo E, Brown R (2003) Systemically administered alpha2-agonistinduced peripheral vasoconstriction in humans. Anesthesiology 99:65-70.

Tononi G (2004) An information integration theory of consciousness. BMC Neurosci 5:42.

Tononi G, Koch C (2008) The neural correlates of consciousness: an update. Ann N Y Acad Sci 1124:239-261

Tu Y, Yu T, Fu XY, Xie P, Lu S, Huang XQ, Gong QY (2011) Altered thalamocortical functional connectivity by propofol anesthesia in rats. Pharmacology 88:322-326.

Xie, G, Deschamps, A, Backman, SB, Fiset, P, Chartrand, D, Dagher, A, Plourde, G (2011) Critical involvement of the thalamus and precuneus during restoration of consciousness with physostigmine in humans during propofol anaesthesia: a positron emission tomography study. Br J Anaesth 106:548 - 557.

Yeganeh MH, Ramzan I (1997) Determination of propofol in rat whole blood and plasma by high-performance liquid chromatography. J Chromatogr B Biomed Sci Appl 691:478-482.

Zhou J, Liu X, Song W, Yang Y, Zhao Z, Ling F, Hudetz AG, Li SJ (2011) Specific and nonspecific thalamocortical functional connectivity in normal and vegetative states. Conscious Cogn 20:257-268. 